\title{
Clinical outcome following lung transplantation in patients with cystic fibrosis colonised with Burkholderia cepacia complex: results from two French centres
}

\author{
V Boussaud, ${ }^{1}$ R Guillemain, ${ }^{2}$ D Grenet, ${ }^{3} \mathrm{~N}$ Coley, ${ }^{4}$ R Souilamas, ${ }^{1}$ P Bonnette, ${ }^{3}$ M Stern ${ }^{3}$
}

\section{See Editorial, p 668, and page 725}

${ }^{1}$ Pôle cardio-thoracique, AP-HP, HEGP, Paris, France;

${ }^{2}$ Département d'AnesthésieRéanimation, AP-HP, HEGP,

Paris, France; ${ }^{3}$ Pôle des Maladies respiratoires, Hôpital Foch, Suresnes, France;

${ }^{4}$ Observatoire Cepacia, Laboratoire de BactériologieHygiène, Institut fédératif de Biologie, Hôpital Purpan,

Toulouse, France

Correspondence to: Dr V Boussaud, Service de chirurgie cardiaque, Hôpital Européen Georges Pompidou, 20-40 rue Leblanc, 75908 Paris Cedex 15, France; veronique. boussaud@egp.aphp.fr

Received 26 August 2007 Accepted 26 March 2008 Published Online First 11 April 2008

\begin{abstract}
Background: Infection with Burkholderia cepacia complex (BCC) is a life threatening complication of cystic fibrosis (CF), often seen as a contraindication for lung transplantation.

Methods: A long term retrospective study was conducted of all patients with CF undergoing lung transplants from January 1990 to October 2006 in two French centres allowing transplantation in patients colonised with BCC.
\end{abstract}

Results: 22 of the 247 lung transplant patients with CF were infected with BCC (B cenocepacia genomovar III $(\mathrm{n}=8), B$ multivorans genomovar $\|(\mathrm{n}=11), B$ vietnamiensis genomovar $\mathrm{V}(\mathrm{n}=2)$ and $B$ stabilis genomovar IV $(n=1))$. BCC colonisation was not associated with any significant excess mortality (HR 1.5, $95 \% \mathrm{Cl} 0.7$ to $3.2 ; \mathrm{p}=0.58$ ). However, early mortality rates tended to be higher in the BCC group than in the non-BCC group (3 month survival: $85 \%$ vs $95 \%$, respectively; $\log$ rank $p=0.05$ ). Univariate analysis showed that the risk of death was significantly higher for the eight patients infected with $B$ cenocepacia than for the other 14 colonised patients (HR 3.2, 95\% Cl 1.1 to $5.9 ; p=0.04)$. None of the other risk factors testedprimary graft failure, late extubation, septicaemia — had a significant effect. The 5 year cumulative incidence rate of bronchiolitis obliterans syndrome was not significantly higher in the BCC group than in the non-BCC group (38\% vs $24 \%$, respectively; $p=0.35$ ).

Conclusion: Our results suggest that BCC infection with a non-genomovar III organism may not be associated with excess mortality after lung transplantation in patients with CF and should not be seen as sufficient reason to exclude lung transplantation. However, colonisation with $B$ cenocepacia remains potentially detrimental.

Cystic fibrosis (CF) is the most common inherited lung disease. It frequently progresses to respiratory failure and death and the treatments currently available aim to slow progression through respiratory therapy, good nutritional support and the treatment of pulmonary exacerbations. In patients with end stage CF, lung transplantation may help to prolong survival and ensure that patients have an acceptable quality of life. Survival rates 1,3 and 5 years after transplantation of $76 \%, 60 \%$ and $49 \%$, respectively, have been reported. ${ }^{1}$ Organ shortages have led some physicians to try to select the "best candidates" for lung transplantation. ${ }^{2}$ One of the criteria used in pretransplantation selection is the absence of infection with Burkholderia cepacia complex (BCC). This gram negative bacterium causes serious opportunistic infections and has emerged as a major pathogen in patients with CF, with the clinical outcome of infection varying from long term bronchial colonisation to life threatening necrotising pneumonia and sepsis, known as "cepacia syndrome". Global mortality rates for BCC colonisation may reach $50 \%,{ }^{3-9}$ depending on genomovar type. However, these rates were determined for patients who had not yet undergone transplantation. Conflicting results have been published concerning post-transplantation outcome. ${ }^{10-14}$ Nonetheless, BCC colonisation remains a contraindication in many transplantation centres worldwide, including the USA, which performs the largest number of transplant operations of any country worldwide.

The purpose of this study was to determine whether patients infected with BCC had a worse outcome after transplantation than those harbouring other common bacteria, to confirm the influence of BCC genomovar and to identify factors predictive of outcome.

\section{METHODS}

\section{Study population}

We retrospectively reviewed the medical records of all patients with CF undergoing lung transplantation at Georges Pompidou European Hospital (HEGP) and Foch Hospital (HF), Paris, France, from January 1990 to October 2006. These centres allow transplantation in BCC colonised patients: all patients identified as being chronically infected with BCC were included in the transplantation programme. These two French centres are responsible for almost all lung transplant procedures in patients with CF and BCC infection in France.

\section{Graft procedure and monitoring}

Lung transplantation procedures were systematically performed under cardiopulmonary bypass (CBP) for patients at HEGP, whereas CPB was carried out selectively in patients at HF. Methylprednisolone was administered during the operative period (500 mg during the preoperative phase then $500 \mathrm{mg}$ during $\mathrm{CPB}$ ), and was subsequently replaced by prednisolone $(1 \mathrm{mg} / \mathrm{kg} /$ day for the first month, with gradual tapering to $5-10 \mathrm{mg} /$ day). Calcineurin inhibitors (ciclosporin or tacrolimus) were initially given intravenously on day 1 , and were then administered orally between days 1 and 8. Daily doses were adjusted to obtain plasma concentrations of between 
200 and $350 \mathrm{ng} / \mathrm{ml}$ for ciclosporin and between 8 and $13 \mathrm{ng} / \mathrm{ml}$ for tacrolimus. Azathioprine was administered at a dose of $1.5-2 \mathrm{mg} /$ $\mathrm{kg} /$ day. After 2000, azathioprine was replaced by mycophenolate at HEGP. Cytolytic induction therapy with rabbit antithymocyte globulin was systematically administered during the first 3 days. Induction therapy was discontinued after 2001 at HF but is still administered at HEGP. Despite the use of these slightly different approaches, the results from the two centres were pooled.

Patients with CF generally harbour bacterial strains with multiple antibiotic resistance. They therefore receive prophylaxis with two or three antibiotics for 10-15 days. All patients colonised with BCC received at least three intravenous antibiotics (beta lactam + aminoside + quinolone) for 10-30 days after surgery, according to postoperative bacteriological documentation. Aerosolised colomycin and tobramycin were administered after extubation. Intravenous implantable devices were systematically removed at the time of surgery for all BCC infected patients. For CMV mismatch patients (donor positive/recipient negative), gancyclovir was administered intravenously at a dose of $5 \mathrm{mg} / \mathrm{kg}$ twice daily and then orally for 3 months. Since 2001, this gancyclovir treatment has been replaced by valgancyclovir ( $900 \mathrm{mg} /$ day) for at least 3 months. Prior or postoperative colonisation with Aspergillus species was treated with itraconazole or voriconazole (after 2003) for up to 6 months. Finally, cotrimoxazole or inhaled pentamidine was administered for an indefinite period after day 14 .

Surveillance bronchoscopy, with bronchoalveolar lavage (BAL) and transbronchial biopsy, was routinely carried out on day 7 and after 1, 3, 6, 9, 12 and 24 months. After 2000, HEGP patients systematically underwent transbronchial biopsy in the first month and thereafter only if acute rejection was suspected. BAL and sputum samples were sent for bacteriological examination and testing for susceptibility to antimicrobial agents.

\section{Data collection}

Epidemiological data on Burkholderia cepacia were obtained from the National Observatory for Cepacia database. All BCC isolates were identified by amplified rDNA restriction analysis. All $B$ cenocepacia genomovar III strains were cbl-negative. These strains, with no cable pili, are different from the ET-12 clone responsible for a North American outbreak. In this group of strains, phylogenetic analysis of the recA cluster revealed a predominance of III-A strains, with only one III-B strain. Clinical data were obtained from the patients' medical records. Follow-up data were obtained from medical charts.

Sputum, BAL fluid and blood samples were systematically cultured, using selective media for BCC. Ceftazidime, tobramycin, ciprofloxacin, aztreonam, imipenem, colomycin and cotrimoxazole were tested, along with other antibiotics, such as chloramphenicol, temocillin, meropenem and doxycycline, if required. BCC was defined as panresistant if no antibiotic was active against the strain, and multiresistant if it was resistant to all beta lactams (including temocillin and meropenem).

Infectious complications, primary graft failure (PGF), incidence of bronchiolitis obliterans syndrome (BOS), survival and causes of death were analysed for all Patients with CF undergoing lung transplantation, and patients with and without BCC infection were compared.

\section{Statistical analysis}

Continuous data were summarised by calculating the mean (SD) or median (25\% percentile; $75 \%$ percentile) as appropriate. Data for recipients with and without BCC colonisation were then compared by univariate analysis. Survival analysis was carried out by plotting Kaplan-Meier curves and comparing distributions by means of the log rank test; Mantel-Haenszel hazard ratios and associated $95 \%$ confidence interval (CI) were calculated. We also estimated the BOS specific cumulative incidence rate, considering death as a competing risk. We

Table 1 Characteristics of patients infected with Burkholderia cepacia complex (BCC)

\begin{tabular}{|c|c|c|c|c|c|c|c|c|c|c|}
\hline $\begin{array}{l}\text { Patient } \\
\text { No }\end{array}$ & BCC & $\begin{array}{l}\text { Age } \\
\text { (y) }\end{array}$ & Sex & Susceptibility & $\begin{array}{l}\text { Induction } \\
\text { therapy }\end{array}$ & $\begin{array}{l}\text { AR } \\
\text { (days) }\end{array}$ & $\begin{array}{l}\text { BO } \\
\text { (months) }\end{array}$ & Status & $\begin{array}{l}\text { Survival } \\
\text { (y) }\end{array}$ & $\begin{array}{l}\text { Cause of } \\
\text { death }\end{array}$ \\
\hline 1 & Vietnamiensis & 22 & $\mathrm{~F}$ & MR & Yes & $\begin{array}{l}14,60,90 \\
150\end{array}$ & No & Alive & 12.1 & - \\
\hline 2 & Vietnamiensis & 17 & $\mathrm{~F}$ & MR & Yes & No & No & Dead & 0.6 & Unknown \\
\hline 3 & Stabilis & 20 & $\mathrm{M}$ & MR & Yes & 6 & No & Alive & 11.5 & - \\
\hline 4 & Multivorans & 24 & $\mathrm{~F}$ & MR & Yes & No & No & Alive & 12.6 & - \\
\hline 5 & Multivorans & 22 & $M$ & MR & Yes & 23,120 & 72 & Alive & 7 & - \\
\hline 6 & Multivorans & 22 & $\mathrm{~F}$ & PR & Yes & No & No & Alive & 6 & - \\
\hline 7 & Multivorans & 24 & $\mathrm{~F}$ & MR & Yes & No & 16 & Alive & 3.6 & - \\
\hline 8 & Multivorans & 21 & $M$ & PR & No & 60 & No & Alive & 1.1 & - \\
\hline 9 & Multivorans & 27 & $\mathrm{~F}$ & PR & Yes & No & No & Alive & 0.5 & - \\
\hline 10 & Multivorans & 20 & $M$ & PR & Yes & 60 & 3 & Dead & 3.8 & BO \\
\hline 11 & Multivorans & 13 & $\mathrm{M}$ & $\mathrm{MR}$ & Yes & $8,90,180$ & 36 & Dead & 3.6 & BO \\
\hline 12 & Multivorans & 22 & $\mathrm{~F}$ & MR & Yes & 9,12 & 7 & Dead & 2 & ARF \\
\hline 13 & Multivorans & 10 & $\mathrm{M}$ & MR & Yes & NA & NA & Dead & 0.8 & ARF \\
\hline 14 & Multivorans & 27 & $\mathrm{~F}$ & $\mathrm{PR}$ & Yes & 54 & 4 & Dead & 0.4 & BO \\
\hline 15 & Cenocepacia & 19 & $\mathrm{M}$ & $\mathrm{PR}$ & No & No & 25 & Alive & 3.8 & - \\
\hline 16 & Cenocepacia & 16 & $\mathrm{M}$ & $\mathrm{PR}$ & No & $5,90,150$ & No & Alive & 0.7 & - \\
\hline 17 & Cenocepacia & 11 & $\mathrm{M}$ & $\mathrm{PR}$ & Yes & 10 & 102 & Dead & 9.9 & Retranspl, MOF \\
\hline 18 & Cenocepacia & 8 & M & MR & Yes & 15,30 & 10 & Dead & 2.2 & BO \\
\hline 19 & Cenocepacia & 20 & $\mathrm{~F}$ & NA & Yes & No & - & Dead & $1 \mathrm{~d}$ & PGF \\
\hline 20 & Cenocepacia & 18 & $\mathrm{~F}$ & MR & No & 9,21 & - & Dead & $34 \mathrm{~d}$ & Cepacia infection \\
\hline 21 & Cenocepacia & 29 & $\mathrm{~F}$ & $\mathrm{PR}$ & Yes & 9 & - & Dead & $92 \mathrm{~d}$ & Cepacia infection \\
\hline 22 & Cenocepacia & 25 & $\mathrm{~F}$ & PR & Yes & No & - & Dead & $59 \mathrm{~d}$ & Cepacia infection \\
\hline
\end{tabular}

AR, acute rejection; ARF, acute respiratory failure; BO, bronchiolitis obliterans; MOF, multiple organ failure; MR, multiresistant; NA, not available; PGF, primary graft failure;

$\mathrm{PR}$, panresistant; Retranspl, retransplantation. 
Table 2 Post-transplantation recurrences of Burkholderia cepacia complex (BCC)

\begin{tabular}{|c|c|c|c|c|c|}
\hline \multirow{2}{*}{$\begin{array}{l}\text { Patient } \\
\text { No }\end{array}$} & \multirow{2}{*}{ BCC } & \multicolumn{2}{|c|}{ Site of positivity after LT } & \multicolumn{2}{|c|}{ Time after LT (days) } \\
\hline & & Lung & BC & Lung & BC \\
\hline 1 & Vietnamiensis & Pos & Neg & $\mathrm{M} 42, \mathrm{Y} 6,4$ & 0 \\
\hline 2 & Vietnamiensis & Pos & Neg & 38,61 & 0 \\
\hline 3 & Stabilis & Pos & Neg & 17,23 & 0 \\
\hline 4 & Multivorans & Pos & Neg & 60 & 0 \\
\hline 5 & Multivorans & Neg & Neg & 0 & 0 \\
\hline 6 & Multivorans & Neg & Neg & 0 & 0 \\
\hline 7 & Multivorans & Pos & Pos & $0-7,22,24$ & $0-5,28$ \\
\hline 8 & Multivorans & Pos & 30 & & \\
\hline 9 & Multivorans & Pos & Neg & 28 & 0 \\
\hline 10 & Multivorans & Pos & Neg & $0-2$ & \\
\hline 11 & Multivorans & Pos & Pos & $1-150$ & 0 \\
\hline 12 & Multivorans & Pos & Pos & 6,9 & 0 \\
\hline 13 & Multivorans & Pos & Neg & $9-39, \mathrm{M} 5, \mathrm{M} 9$ & 0 \\
\hline 14 & Multivorans & Pos & Pos & 11 & 0 \\
\hline 15 & Cenocepacia & Pos & Neg & 5 & 0 \\
\hline 16 & Cenocepacia & - & - & & \\
\hline 17 & Cenocepacia & NA & NA & 0 & 0 \\
\hline 18 & Cenocepacia & Pos & Pos & $1-24$ & $0-33$ \\
\hline 19 & Cenocepacia & Pos & Pos & $4-9,43,69$ & 35 \\
\hline 20 & Cenocepacia & Pos & Pos & $46,48-92,94$ & 0 \\
\hline 21 & Cenocepacia & Pos & Pos & $1-59$ & $1-59$ \\
\hline 22 & Cenocepacia & $\mathrm{Neg}$ & Neg & 0 & 0 \\
\hline
\end{tabular}

$\mathrm{BC}$, blood culture; $\mathrm{LT}$, lung transplantation; NA, not available.

assessed the equivalence of subdistributions between patients with and without BCC colonisation, using Gray's test. Statistical analysis was carried out with Graphdpad and R software. A p value less than 0.05 was considered statistically significant.

\section{RESULTS}

\section{Demographics}

Between January 1990 and October 2006, 495 patients underwent lung transplantation ${ }^{15}$; 247 of these patients had CF (126 men and 121 women, mean age 21.5 (7.7) years) and were included in the study. Surgical procedures were as follows: 213 bilateral lung transplantations, 24 combined lung-liver, six combined heart-lung-liver and four heart-lung transplant procedures. There were nine retransplantation procedures: seven for BOS and two for PGF.

Twenty-two of the 247 patients with CF (8.9\%) were chronically infected with BCC before transplantation, with a mean age at first colonisation of 18 (4) years. One patient was infected with Burkholderia gladioli, which is not part of the Burkholderia cepacia complex, and was therefore excluded from the BCC population. Mean age was similar in the BCC and nonBCC groups (20 (6) vs 22 (8) years, respectively; $p=0.32$ ).

The 22 BCC colonised patients comprised 12 women and 10 men. Eleven patients were infected with $B$ cepacia multivorans genomovar II, eight with $B$ cepacia cenocepacia genomovar III and the remaining three were infected with other species of Burkholderia: $B$ vietnamiensis in two patients and $B$ stabilis in the third. Antibiograms were available in 21 cases: 11 strains were multiresistant and 10 were panresistant.

The characteristics of the patients in the BCC group are summarised in table 1.

All patients underwent double lung transplant procedures, except patient No 4 who underwent combined lung-liver transplantation.

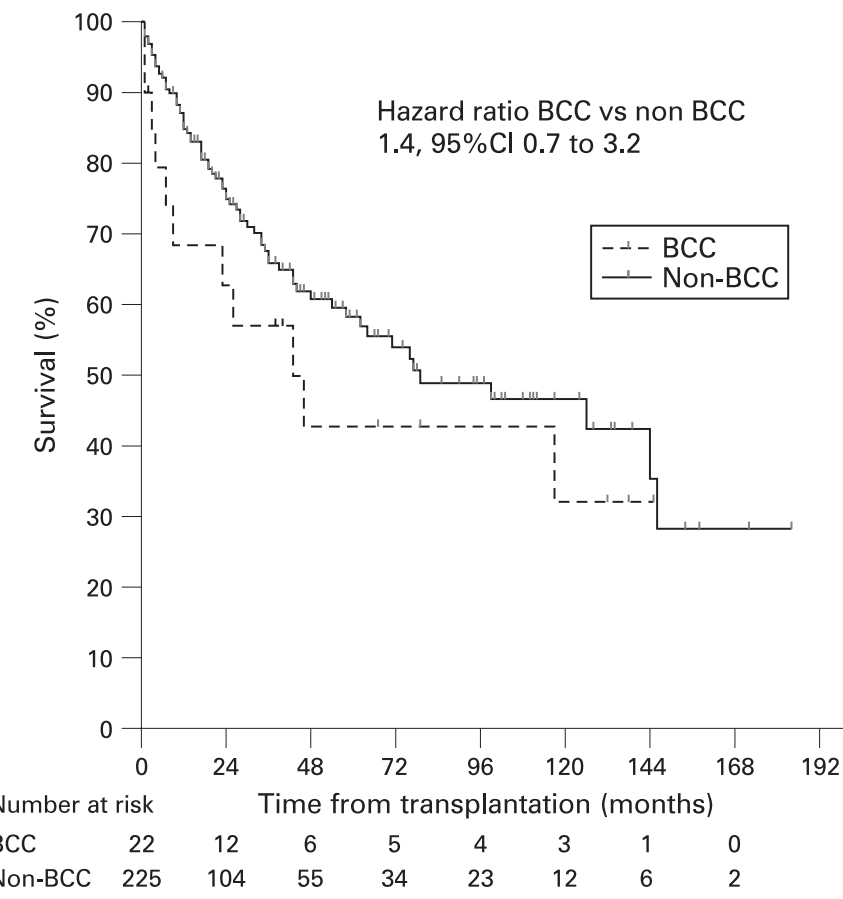

Figure 1 Survival of patients infected with Burkholderia cepacia complex (BCC).

\section{Infection after lung transplantation}

No patient without BCC infection before lung transplantation developed infection after transplantation. In 17 of the 22 BCC positive patients, postoperative pulmonary specimens (sputum or BAL) tested positive for BCC. Three of these patients died of extensive necrotising pneumonia within 3 months of transplantation (table 2). These three patients were all infected with $B$ cenocepacia.

One of the four patients with positive blood cultures had $B$ multivorans septicaemia originating from an implantable device. This infection resolved after removal of the device. The patient died 4 months after transplantation, from early BOS. Two patients developed uncontrollable $B$ cenocepacia sepsis and died in intensive care within 2 months of transplantation. One patient developed $B$ cenocepacia empyema after 1 month and recovered after surgical treatment. All patients with recurrences of BCC colonisation were treated only if infection was confirmed by clinical, radiological and bacteriological data.

\section{Outcome}

In total, 109 recipients with CF died. There were 97 deaths in the non-BCC group and 12 in the BCC group. Three of the 12 deaths in the BCC group were directly related to BCC whereas the other nine were caused by PGF in one case, unknown causes (7 months after transplantation) in one case, complications of BOS in five cases and acute respiratory failure, 10 months and 2 years after transplantation, in two cases (table 1). Median survival time was 79 months for non-BCC patients and 43 months for BCC patients. Overall mortality risk was similar in the two groups (hazard ratio (HR) for death of BCC patients vs non-BCC patients 1.4 (95\% CI 0.7 to 3.2); log rank test $\mathrm{p}=0.58)$ but early mortality rates tended to be higher in the BCC group (3 month survival $85 \%$ in the BCC group vs $95 \%$ in the non-BCC group; log rank $p=0.05$ ).

One patient in the BCC group died during surgery, eight of 12 patients were weaned from mechanical ventilation before day 3 


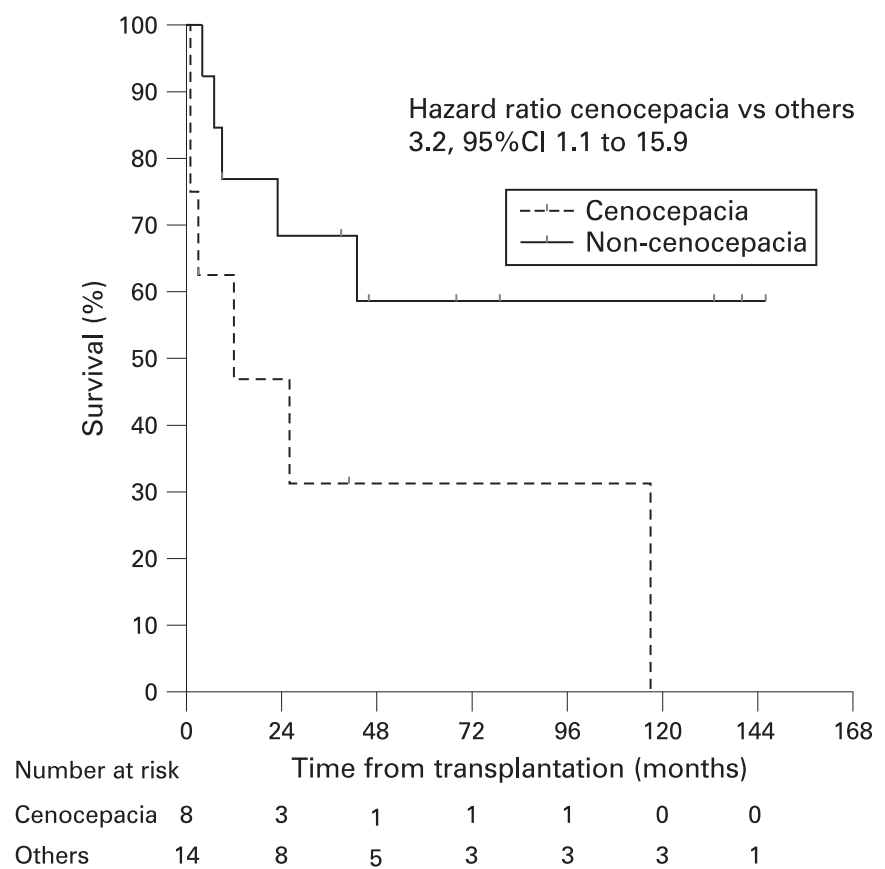

Figure 2 Survival of patients infected with Burkholderia cepacia cenocepacia.

whereas this was the case for only two of 10 patients who underwent late extubation or tracheotomy. Six of seven patients who developed grade 3 PGF eventually died compared with only six of the 15 who did not. One of the four patients in the BCC group who did not receive induction therapy died, compared with 11 of the 18 patients who did.

\section{Specificity of $\boldsymbol{B}$ cenocepacia genomovar III}

Patients infected with $B$ cenocepacia $(n=8)$ had higher mortality rates than patients infected with other strains of BCC $(n=14)$ (HR for death of cenocepacia infected patients vs others 3.2 (95\% CI 1.1 to 15.9 ); $p=0.04$ ) (figs 1,2 ). Six of the eight patients died, with three of these deaths directly linked to $B$ cenocepacia infection, during the postoperative period. Surgery was difficult in two of these patients. One patient had a thoracic malformation causing technical problems, suffered bleeding and received a graft from a borderline donor with condensation of the left lower lobe, leading to grade 4 PGF. She developed immediate pulmonary and pleural infection and septicaemia refractory to combinations of multiple antibiotics. The second patient suffered from cardiac arrest, air embolism, severe pulmonary hypertension and grade 4 PGF. She developed pulmonary opacities, pleural effusion and septicaemia within 10 days of transplantation. The third patient suffered from grade 3 PGF, underwent tracheotomy because of phrenic paralysis and developed non-septicaemic pulmonary cepacia infection that progressed despite combined antibiotic treatment.

\section{BOS}

We analysed the association between BCC and BOS in the 212 patients of the cohort alive 90 days after transplantation. Sixty of these patients developed BOS. If death was considered as a competing event, the 5 year BOS specific cumulative incidence rate was $38 \%$ for the BCC group compared with $24 \%$ for the non-BCC group $(p=0.35)$.

\section{DISCUSSION}

Chronic infection with multiresistant BCC is associated with poor outcome in patients with $\mathrm{CF}^{5}{ }^{16}$ and may represent a threat to recipients on high doses of immunosuppressive treatment. This large study suggests that patients infected with BCC are at no greater risk than uninfected patients of death following lung transplantation. Excess mortality was observed in the genomovar III subgroup with respect to genomovars II, IV and V. Nonetheless, some patients in this subgroup survived for prolonged periods and died from causes other than cepacia complications (one patient died after nearly 10 years from multiple organ failure after retransplantation). However, this study is subject to several limitations: differences in mortality between BCC and non-BCC patients must be interpreted with caution because of the small number of patients in the BCC group; we cannot exclude the possibility that our study has insufficient power. Multivariate analysis was not possible. In addition, the two centres differed in terms of the treatment given, particularly with regard to cytolytic induction therapy and the duration of antibiotic treatment. Furthermore, 90 day mortality rates in the non-BCC group were particularly high because the study was retrospective and included data from the first lung transplantation procedures for which postoperative mortality rates were particularly high.

The prevalence of BCC infection is 3\% in France ${ }^{17}$ and $3.1 \%$ in North America. ${ }^{18}$ However, some regions of North America have a particularly high prevalence, reaching $20 \%$ in the USA and $13 \%$ in Canada. ${ }^{19}$ Revisions of Burkholderia taxonomy have led to the definition of nine different species, the virulence and clinical impact of which remain unclear. B cenocepacia (genomovar III) accounts for more than half of all isolates worldwide- $83 \%$ of all isolates in Canada, ${ }^{20} 50 \%$ in the USA ${ }^{21}$ and the $\mathrm{UK}^{13}$ - and has been associated with higher mortality and morbidity. B multivorans (genomovar II) accounts for one-third of US isolates and is believed to be less virulent. ${ }^{22}$ In France, $16 \mathrm{~S}$ rRNA analysis has shown these genomovars to have similar prevalences $^{23}: 49.5 \%$ for genomovar III and $41.1 \%$ for genomovar II.

Several putative virulence factors other than genomovar have been identified: expression of a cable-like pilus, ${ }^{24}$ interleukin 8 stimulation $^{25}$ and proinflammatory activity, ${ }^{26}$ production of haemolytic toxin, ${ }^{27}$ proteases, ${ }^{28}$ siderophores ${ }^{29}$ and escape from host cell microbicidal activity by means of delayed phagosome maturation..$^{30}$ The promotion of biofilm development may also increase the resistance and tolerance of BCC to antibiotics. ${ }^{31}$ Several studies have investigated the impact of Burkholderia cepacia, with conflicting results. In 1993, Snell et al reported their results for patients with CF with positive cultures for $B$ cepacia ${ }^{10}$ : $85 \%$ of such patients died from $B$ cepacia pneumonia within 2 months of transplantation. UK centres gave more promising results, with no differences in mortality observed..$^{11}$ In Toronto, where the incidence of $B$ cepacia is high (52\% of the 53 patients undergoing transplantation), Chaparro et al found evidence for excess mortality (65\% vs $36 \%$ ) within 3 months of transplantation ( $60 \%$ of all deaths) and an excess risk of BOS. ${ }^{14}$ These findings were consistent with other North American data $^{32}$ establishing a link with genomovar type. The work of De Soyza et al supported these findings, ${ }^{12}$ with $100 \%$ mortality observed in patients infected with $B$ cenocepacia (genomovar III). In France, 120 patients were recorded on the Observatory for Cepacia register in 2004. ${ }^{17}$ Mean age at first colonisation was 14.7 (7.9) years, with a higher proportion of men (70 vs 50), particularly for $B$ cenocepacia isolates (genomovar III). Three cases of spontaneous septicaemia were observed, with one death 
within a year. BCC mortality rates remain low in the French CF population, with three deaths in 2004, a figure comparable with the postoperative results reported here for lung transplant patients. However, long term survival was also recorded in some members of this population, which is encouraging. Prolonged treatment with a combination of antibiotics seems to be a reasonable approach to the treatment of these patients. ${ }^{14} \mathrm{We}$ frequently used a combination of antibiotics (ceftazidime or temocillin or meronem + tobramycin + ciprofloxacin), particularly in cases of panresistant bacteria, and no BCC infection was observed other than in the three patients with $B$ cenocepacia. Cotrimoxazole or chloramphenicol could also be used if the strains were sensitive. Triple associations are known to be effective against BCC. ${ }^{33}$ However, the question of combination therapy against multiresistant or panresistant organisms in CF has recently been addressed: Aaron et al demonstrated that antibiotic treatment directed by susceptibility testing for combinations of antibodies gave no improvement in clinical and bacteriological outcomes over treatment directed by standard culture and susceptibility techniques. ${ }^{34}$ Little is known about the non-bactericidal effect of antibiotic treatment, but such effects may play an important role in determining the improvement of patients with $\mathrm{CF}$, particularly in terms of susceptibility to Pseudomonas or $B$ cepacia infections. Greater insight into inflammatory trigger receptors might help to increase our understanding of defences against bacteria. ${ }^{35}$

Liou et al recently refined the selection of potential candidates for lung transplantation. ${ }^{2}$ This statistical analysis of the UNOS (United Network for Organ Sharing) database and the CFFP Registry (CF Foundation Patient Registry) suggest that the outlook is gloomy for BCC infected patients, but this analysis did not distinguish between genomovars. The authors suggested the restriction of lung transplantation in BCC infected patients, despite the improvements in quality of life and survival provided by such treatment. However, we feel that extending lifespan is not the only relevant goal. CF is the leading indication for lung transplantation in France, accounting for $35.8 \%$ of all cases, followed by emphysema and chronic obstructive pulmonary disease (19.8\%). The benefits of transplantation for patients with chronic obstructive pulmonary disease, in terms of prolonged survival, have not been clearly demonstrated, ${ }^{36}$ but no one would question the validity of this indication. Physical and psychological functioning is also a key issue for patients with CF who have had to cope with overwhelming frustration for years, particularly in cases of BCC colonisation that might lead to the patient being excluded from a transplantation programme. Survival is clearly a matter of concern, but we feel that decisions relating to the allocation of resources for lung transplantation should not be restricted to this dimension. Changes in the organisation of organ procurement in France, with re-evaluation of existing lung donor pool, have led to a $100 \%$ increase in the number of organs available over the past 3 years. ${ }^{37}$ The number of organs offered for transplantation has clearly increased since transplantation teams have been allowed a role in selection, leading to the use of donor lungs that would previously have been refused. The time spent on the waiting list for transplantation has been cut back significantly, to 6 months. The discrepancy between the number of organs donated and the number of potential recipients has decreased considerably, and the number of deaths on the waiting list has also decreased, from $16.1 \%$ in 2000 to $7.2 \%$ in $2005 .{ }^{37}$ This increase in organ allocation does not necessarily mean that we can ignore previous contraindifcations for lung transplantation. However, although some transplantation centres are still unwilling to carry out lung transplantation on patients with BCC, our results suggest that colonisation with a non-genomovar III species does not necessarily increase the risk of a poor outcome. Even infection with BCC genomovar III should not necessarily be seen as a contraindication for lung transplantation, although our results suggest that such infection may be associated with a higher risk. Although more than $30 \%$ of these transplantations were unsuccessful, the majority could be considered successful, at least in the short term. We feel that it is important to give these patients a chance. For the time being, it is not possible to identify those patients infected with genomovar III most likely to benefit from transplantation. Further studies comparing the results obtained in different countries should be conducted, making it possible to carry out a multivariate analysis to identify potential risk factors for infection with these organisms and to determine the utility of prolonged antibiotic treatment or pleural washing.

Our experience suggests that patients with CF infected with BCC of non-cenocepacia type should not be denied access to transplantation. However, early mortality rates are higher in cases of $B$ cenocepacia genomovar III colonisation, so patients and their relatives should be informed of the higher risk of an unfavourable outcome.

Acknowledgements: The authors wish to acknowledge the assistance provided by Ludovic Trinquart (Epidemiology and clinical research, INSERM CIE4, HEGP, Paris), Clément Picard, Christine Segonds and Isabelle Sermet-Gaudelus in reviewing the manuscript and all the members of Anaesthesiology and Intensive Care Unit in Foch Hospital and HEGP for their dedication to patient care.

Competing interests: None.

\section{REFERENCES}

1. Trulock EP, Edwards LB, Taylor DO, et al. Registry of the International Society for Heart and Lung Transplantation: twenty-third official adult lung and heart-lung transplantation report-2006. J Heart Lung Transplant 2006;25:880-92.

2. Liou TG, Adler FR, Huang D. Use of lung transplantation survival models to refine patient selection in cystic fibrosis. Am J Respir Crit Care Med 2005;171:1053-9.

3. Govan JR, Deretic V. Microbial pathogenesis in cystic fibrosis: mucoid Pseudomonas aeruginosa and Burkholderia cepacia. Microbiol Rev 1996;60:539-74.

4. Muhdi K, Edenborough FP, Gumery L, et al. Outcome for patients colonised with Burkholderia cepacia in a Birmingham adult cystic fibrosis clinic and the end of an epidemic. Thorax 1996;51:374-7.

5. Corey M, Farewell V. Determinants of mortality from cystic fibrosis in Canada, 19701989. Am J Epidemiol 1996;143:1007-17.

6. Whiteford ML, Wilkinson JD, McColl JH, et al. Outcome of Burkholderia (Pseudomonas) cepacia colonisation in children with cystic fibrosis following a hospital outbreak. Thorax 1995;50:1194-8.

7. Frangolias DD, Mahenthiralingam E, Rae S, et al. Burkholderia cepacia in cystic fibrosis. Variable disease course. Am J Respir Crit Care Med 1999;160(5 Pt 1):1572-7.

8. Ledson MJ, Gallagher MJ, Jackson M, et al. Outcome of Burkholderia cepacia colonisation in an adult cystic fibrosis centre. Thorax 2002;57:142-5.

9. Fitzgerald DA, Cooper DM, Paul M, et al. Burkholderia cepacia in cystic fibrosis: novel Australian cluster strain without accelerated respiratory deterioration. J Paediatr Child Health 2001;37:130-6.

10. Snell GI, de Hoyos A, Krajden M, et al. Pseudomonas cepacia in lung transplant recipients with cystic fibrosis. Chest 1993;103:466-71.

11. Egan JJ, McNeil K, Bookless B, et al. Post-transplantation survival of cystic fibrosis patients infected with Pseudomonas cepacia. Lancet 1994;344:552-3.

12. De Soyza A, McDowell A, Archer L, et al. Burkholderia cepacia complex genomovars and pulmonary transplantation outcomes in patients with cystic fibrosis. Lancet 2001;358:1780-1.

13. De Soyza A, Morris K, McDowell A, et al. Prevalence and clonality of Burkholderia cepacia complex genomovars in UK patients with cystic fibrosis referred for lung transplantation. Thorax 2004;59:526-8.

14. Chaparro C, Maurer J, Gutierrez C, et al. Infection with Burkholderia cepacia in cystic fibrosis: outcome following lung transplantation. Am J Respir Crit Care Med 2001;:163:43-8.

15. Kalish LA, Waltz DA, Dovey $M$, et al. Impact of Burkholderia dolosa on lung function and survival in cystic fibrosis. Am J Respir Crit Care Med 2006;173:421-5.

16. Liou TG, Adler FR, Fitzsimmons SC, et al. Predictive 5-year survivorship model of cystic fibrosis. Am J Epidemiol 2001;153:345-52.

17. Chabanon G, Segonds C, Clavel-Batut P, et al. Burkholderia et bactéries apparentées: rapport épidémiologique 2004. Toulouse: Observatoire Cepacia, Laboratoire de bactériologie-Hygiène, Institut fédératif de biologie, Hôpital PurpanToulouse, 2004 
18. Cystic Fibrosis Foundation. Patient Registry 2005 Annual Report. Bethesda: Cystic Fibrosis Foundation, 2006.

19. Mahenthiralingam E, Vandamme P, Campbell ME, et al. Infection with Burkholderia cepacia complex genomovars in patients with cystic fibrosis: virulent transmissible strains of genomovar III can replace Burkholderia multivorans. Clin Infect Dis 2001;33:1469-75.

20. Speert DP, Henry D, Vandamme P, et al. Epidemiology of Burkholderia cepacia complex in patients with cystic fibrosis, Canada. Emerg Infect Dis 2002;8:181-7.

21. LiPuma JJ, Spilker T, Gill LH, et al. Disproportionate distribution of Burkholderia cepacia complex species and transmissibility markers in cystic fibrosis. Am J Respir Crit Care Med 2001;164:92-6.

22. Jones AM, Dodd ME, Govan JR, et al. Burkholderia cenocepacia and Burkholderia multivorans: influence on survival in cystic fibrosis. Thorax 2004; 59:948-51.

23. Segonds C, Heulin T, Marty N, et al. Differentiation of Burkholderia species by PCRrestriction fragment length polymorphism analysis of the 16S rRNA gene and application to cystic fibrosis isolates. J Clin Microbiol 1999;37:2201-8.

24. Sun L, Jiang RZ, Steinbach S, et al. The emergence of a highly transmissible lineage of cbl+ Pseudomonas (Burkholderia) cepacia causing CF centre epidemics in North America and Britain. Nat Med 1995;1:661-6.

25. Palfreyman RW, Watson ML, Eden C, et al. Induction of biologically active interleukin-8 from lung epithelial cells by Burkholderia (Pseudomonas) cepacia products. Infect Immun 1997;65:617-22.

26. De Soyza A, Ellis CD, Khan CM, et al. Burkholderia cenocepacia lipopolysaccharide, lipid A, and proinflammatory activity. Am J Respir Crit Care Med 2004;170:70-7.
27. Hutchison ML, Poxton IR, Govan JR. Burkholderia cepacia produces a hemolysin that is capable of inducing apoptosis and degranulation of mammalian phagocytes. Infect Immun 1998;66:2033-9.

28. Kooi C, Cox A, Darling P, et al. Neutralizing monoclonal antibodies to an extracellular Pseudomonas cepacia protease. Infect Immun 1994;62:2811-17.

29. McKenney D, Allison DG. Effects of growth rate and nutrient limitation on virulence factor production in Burkholderia cepacia. J Bacteriol 1995:177:4140-3.

30. Lamothe J, Huynh KK, Grinstein S, et al. Intracellular survival of Burkholderia cenocepacia in macrophages is associated with a delay in the maturation of bacteriacontaining vacuoles. Cell Microbiol 2007:9:40-53.

31. Greenberg EP. Bacterial communication: tiny teamwork. Nature 2003:424:134.

32. Aris RM, Routh JC, LiPuma JJ, et al. Lung transplantation for cystic fibrosis patients with Burkholderia cepacia complex. Survival linked to genomovar type. Am J Respir Crit Care Med 2001;164:2102-6.

33. Aaron SD, Ferris W, Henry DA, et al. Multiple combination bactericidal antibiotic testing for patients with cystic fibrosis infected with Burkholderia cepacia. Am J Respir Crit Care Med 2000;161:1206-12.

34. Aaron SD, Vandemheen KL, Ferris W, et al. Combination antibiotic susceptibility testing to treat exacerbations of cystic fibrosis associated with multiresistant bacteria: a randomised, double-blind, controlled clinical trial. Lancet 2005;366:463-71.

35. Munford RS, Varley AW. Shield as signal: lipopolysaccharides and the evolution of immunity to gram-negative bacteria. PLoS Pathog 2006;2:e67.

36. Hosenpud JD, Bennett LE, Keck BM, et al. Effect of diagnosis on survival benefit of lung transplantation for end-stage lung disease. Lancet 1998;351:24-7.

37. Camby C. Rapport annuel 2005 - Bilan de activités de l'agence de la biomédecine. Agence de la Biomédecine, 2005:111-15.

\section{Lung alert}

\section{Early routine follow-up chest radiographs for pneumonia are not useful}

This study investigated the clinical value of chest radiographs during the 4-week period after diagnosis of severe community acquired pneumonia (CAP) in immune competent hospitalised patients. The time to resolution of pulmonary infiltrates and other radiographic abnormalities caused by infection was compared with resolution of clinical parameters, and factors associated with delayed resolution were identified.

Two hundred and eighty-eight hospitalised patients were followed up between July 2000 and June 2003. Severe pneumonia was defined as a pneumonia severity index score of $>90$ or according to the American Thoracic Society definition. Patients were treated according to the Dutch guidelines for CAP management. The time for clearance of chest radiographic abnormalities was estimated from chest radiographs taken at 0,7 and 28 days. Clearance of chest radiographic abnormalities lagged considerably behind clinical improvement at all the assessment time intervals. Raised C-reactive protein levels $>200 \mathrm{mg} / \mathrm{dl}$, multilobar infiltrates, dullness on percussion, raised urea levels and Streptomycin pneumoniae infection on admission were independent factors associated with delayed radiographic resolution. Age and comorbidities did not influence the rate of radiographic clearance.

This study was based on secondary data from a prospective randomised trial on the costeffectiveness of an early switch from parenteral to oral treatment for severe CAP, which may not be the standard clinical practice in many settings. However, it addressed a pertinent question and might influence the way physicians manage CAP.

- Burns AHW, Oosterheert JJ, Prokop M, et al. Patterns of resolution of chest radiograph abnormalities in adults hospitalized with severe community-acquired pneumonia. Clin Infect Dis 2007;45:983-91.

\section{J Sembatya}

Correspondence to: Dr J Sembatya, Clinical Fellow, Department of Respiratory Medicine, Homerton Hospital, London E9 6SR, UK; ssembi_k@yahoo.com 